Draft version November 20, 2018

Preprint typeset using $\mathrm{LAT}_{\mathrm{E}} \mathrm{X}$ style emulateapj v. 11/10/09

\title{
RADIO ASTROMETRY OF THE TRIPLE SYSTEMS ALGOL AND UX ARIETIS
}

\author{
W. M. Peterson, R. L. Mutel \\ Department of Physics and Astronomy, University of Iowa, Van Allen Hall, Iowa City, Iowa 52242, USA
}

J.-F. LESTRADE

C.N.R.S., Observatoire de Paris, F-75014 Paris, FR

M. GÜDEL

Department Astronomy, University of Vienna, Vienna, AT

W. M. Goss

National Radio Astronomy Observatory, Socorro, NM, USA 87801

Draft version November 20, 2018

\begin{abstract}
We have used multi-epoch long-baseline radio interferometry to determine the proper motion and orbital elements of Algol and UX Arietis, two radio-bright, close binary stellar systems with distant tertiary components. For Algol, we refine the proper motion and outer orbit solutions, confirming the recent result of Zavala et al. (2010) that the inner orbit is retrograde. The radio centroid closely tracks the motion of the KIV secondary. In addition, the radio morphology varies from double-lobed at low flux level to crescent-shaped during active periods. These results are most easily interpreted as synchrotron emission from a large, co-rotating meridional loop centered on the K-star. If this is correct, it provides a radio-optical frame tie candidate with an uncertainty \pm 0.5 mas. For UX Arietis, we find a outer orbit solution that accounts for previous VLBI observations of an acceleration term in the proper motion fit. The outer orbit solution is also consistent with previously published radial velocity curves and speckle observations of a third body. The derived tertiary mass, 0.75 solar masses, is consistent with the K1 main-sequence star detected spectroscopically. The inner orbit solution favors radio emission from the active K0IV primary only. The radio morphology, consisting of a single, partially resolved emission region, may be associated with the persistent polar spot observed using Doppler imaging.

Subject headings: astrometry, stellar dynamics, binaries:close, radio continuum:stars
\end{abstract}

\section{INTRODUCTION}

Multi-epoch phase-referenced radio interferometry is a powerful method to determine astrometric properties of radio-loud objects in a variety of astrophysical settings. Very long baseline interferometry (VLBI), with baselines exceeding $10,000 \mathrm{~km}$, can provide astrometric accuracies between 10 and $100 \mu$ as (Pradel et al. 2006), allowing direct distance measurements to many galactic and extragalactic radio sources with unprecedented accuracy using trigonometric parallax (e.g., Reid et al. 2009, Deller et al. 2009: Dzib et al. 2010). Other astrometric VLBI applications include tests of general relativity (Fomalont et al. 2009), precise determination of Earth's inertial trame (Petrov 2007; Petrov et al. 2009), and searches for extra-solar planets in late-type stellar systems (Bower et al. 2009).

In this paper, we report on astrometric VLBI studies of two well-studied active stellar systems, Algol and UX Arietis. Both systems contain a very active $\mathrm{K}$ sub-giant tidally locked in a short period binary system with a distant third companion. In addition, both are relatively close $(\leq 50 \mathrm{pc})$, so the angular scale of the inner orbit is several times the resolving beam of a global VLBI array. This allows tracking the motion of the active component within the inner binary orbit. By imaging the radio-loud component over many epochs, it is possible to determine all astrometric parameters (parallax, proper motion, inner and outer orbital elements) with high accuracy. In addition, the resulting maps provide a direct probe of the geometry of the coronal structure. Finally, if the solution is sufficiently accurate, the position of active star can be inferred on the radio image, allowing a tie between the optical reference frame and the radio ICRF with sub-mas accuracy (Lestrade et al. 1999; Bourda et al. 2010).

Algol (HD 19356, B8V+K2IV, $29 \mathrm{pc}$ ), the prototype of the eponymous Algol binary class, has been wellstudied since its identification as an eclipsing binary system more than two centuries ago (Goodricke 1783). The cooler subgiant secondary fills its Roche lobe, causing episodic mass transfer onto an accretion disk surrounding the main sequence primary (Richards \& Albright 1993). The subgiant is chromospherically and coronally active, with frequent strong flares observed at radio (e.g., $\mathrm{Mu}-$ tel et al. 1998; Richards et al. 2003), ultraviolet (e.g., Stern et al. 1995), and X-ray (e.g., Ottmann \& Schmitt 1996: Schmitt \& Favata 1999) wavelengths. The close binary pair has an orbital period of 2.83 days and is tidally locked. A distant tertiary companion (F1V, $\mathrm{P}=1.86 \mathrm{yr}$ ) is in an eccentric orbit oriented nearly perpendicular to the inner binary orbit. 
Although most of Algol's orbital parameters for both inner and outer orbits were well-determined spectroscopically decades ago (Hill et al. 1971; Bachmann \& Hershey 1975 Stein \& Beardsley 1977), several parameters were either undetermined or ambiguous until more recent direct imaging became possible. The inner binary's orientation was first determined by Lestrade et al. (1993) who observed the active K-star's radio emission at both quadratures using a global VLBI array. Pan et al. (1993), using optical interferometric data, determined that the orientation of the outer orbit was nearly perpendicular to the inner orbit, confirming the position angle inferred from speckle data (Bonneau 1979).

Determination of the inclination of the inner orbital plane has proved more problematic. Csizmadia et al. (2009) imaged Algol using both an optical interferometer and VLBI. They found that the inner orbit was prograde, i.e. that the line-of-sight component of the orbital angular momentum pointed toward the observer. However Zavala et al. (2010) imaged both the inner and outer binaries at multiple epochs using the NPOI 6-element interferometer and found that the inner orbit is retrograde. In addition, they found that Pan et al. (1993)'s determination of the outer orbit ascending node longitude was in error by $180^{\circ}$.

Interpreting the physical properties of a stellar radio source critically depends on accurately registering the radio morphology with its location within the stellar system. Algol was the first stellar system ever imaged using VLBI (Clark et al. 1975). They found that during an exceptionally large radio flare $(S \sim 600 \mathrm{mJy})$, the source characteristic size was comparable to the overall angular size of the inner binary system. The inferred high brightness temperature and broad spectrum were consistent with gyrosynchrotron emission from mildly relativistic electrons in a coronal magnetic field. Subsequent VLBI images (Mutel et al. 1985, Lestrade et al. 1988; Mutel et al. 1998; Massi et al. 2002; Csizmadia et al. 2009) confirmed this basic picture, but the limited angular resolution and astrometric accuracy of these observations taken separately prevented an accurate registration with the stellar components. However, recently Peterson et al. (2010) observed Algol at six epochs using a high-sensitivity global VLBI array at a higher frequency $(15 \mathrm{GHz})$ than past observations. These data allowed much better sensitivity and angular resolution. They found that the radio structure consisted of a large coronal loop that was centered on the active $\mathrm{K}$ star, aligned along the inner binary axis, and co-rotating. In this paper we combine this dataset with archival phasedreferenced VLBI observations made since 1983. This combined dataset allows a global solution for all astrometric parameters.

UX Arietis (HD 21242, G5V+K0IV, $50 \mathrm{pc}$ ) is an active double-lined spectroscopic binary system. The primary $\mathrm{K}$ subgiant shows activity at radio through UV wavelengths very similar to Algol (Carlos \& Popper 1971; Trigilio et al. 1998; Mutel et al. 1998; Torricelli-Ciamponi et al.|1998, Lang \& Willson 1988; Buccino \& Mauas 2009; Ekmekci 2010). There is also chromospheric activity associated with the secondary, possibly caused by episodic mass-transfer associated with Roche-lobe overflow from the primary (Huenemoerder et al. 1989, Gu et al. 2002; Aarum Ulvås \& Engvold 2003a; Ekmekci 2010). Vogt \&
Hatzes (1991) used Doppler imaging to detect a large, stable polar spot on the $\mathrm{K}$ primary, as well as transient spots at intermediate latitudes, which appear to be preferentially oriented toward the G companion (Rosario et al. 2008). As with Algol, VLBI imaging of the radio corona of UX Arieties (Mutel et al. 1984, Beasley \& Güdel 2000, Massi et al. 2002) shows structure comparable to the inner binary separation and a brightness temperature consistent with gyrosynchrotron emission.

A third component has long been suspected in the UX Arietis system, based on both radial velocity anomalies (Duemmler \& Aarum 2001, Glazunova et al. 2008), speckle observations of a distant third body (e.g., $\overline{M c A l}-$ ister et al. 1987, Hartkopf et al. 2000, Balega et al. 2006), and apparent acceleration in proper motion studies (Lestrade et al. 1999, Boboltz et al. 2003, Fey et al. 2006). In this paper, we combine new multi-epoch VLBI measurements with archival VLBI data to solve for all astrometric components including a tertiary component in a long-period orbit.

\section{OBSERVATIONS}

Algol and UX Arietis were observed with a global VLBI array at six and four epochs respectively between June 2008 and October 2009. The array comprised ten $25 \mathrm{~m}$ telescopes of the Very Long Baseline Array (VLBA) the $100 \mathrm{~m}$ Green Bank Telescope (GBT), and the $100 \mathrm{~m}$ Effelsberg telescope (EB). Observations were performed at $15.4 \mathrm{GHz}$ in dual-polarization mode with a bandwidth of $128 \mathrm{MHz}$ in each polarization. The array synthesized beamsize $(\sim 0.5$ mas $)$ was considerably smaller than the projected angular separation of the inner binary orbit of either system, so we were able to map motion within the inner binary. In addition, we used both previously published and archival VLBA observations to supplement our data, allowing highly accurate global astrometric solutions over a time internal spanning more than 25 years.

We used the 'nodding' phase-referencing technique (Lestrade et al. 1990; Beasley \& Conway 1995, Fomalont et al. 1995), switching rapidly between a compact extragalactic ICRF source with well-determined coordinates (Ma et al. 1998) and the target star. For the Algol observations, we used two phase calibrators: The angularly close primary phase calibrator ICRF source J031301.9+41200 $\left(\Delta \theta=1.0^{\circ}\right)$ and a secondary calibrator, J031049.8+381453 $\left(\Delta \theta=2.8^{\circ}\right)$. We alternated between two-minute scans on Algol and one-minute scans on the primary phase calibrator, with additional oneminute scans of the secondary calibrator every hour. For the UX Arietis observations, we alternated between scans of UX Arietis and ICRF source J033630.1+321829 (a.k.a. NRAO $140, \Delta \theta=4.2^{\circ}$ ) using a 90 -second cycling time.

In addition to these observations, we included two other radio astrometric measurements for these stars. First, we included several previously published radio interferometer positions (Lestrade et al. 1993, 1999, Boboltz et al. 2003, Fey et al. 2006). Second, a number of phase-referenced observations of both Algol and UX Arietis exist in the NRAO VLBA archive (Sjouwerman et al. 2004). We calibrated and imaged these data

1 The National Radio Astronomy Observatory is operated by Associated Universities Inc., under cooperative agreement with the National Science Foundation. 
and present the resulting positions in this paper for the first time. All VLBI data analyzed for this paper are summarized in Table 1

\section{ANALYSIS}

\subsection{Data Calibration and Imaging}

VLBI visibilities from all epochs were calibrated and imaged using the NRAO AIPS software package (Greisen 2003), except for the 1983.5-1994.4 UX Arietis positions that were analysed using the SPRINT software package (cf. Lestrade et al. 1999). We followed standard VLBI amplitude and delay/rate calibration procedures. Geometric delays introduced by small errors in the predicted values of the Earth Orientation Parameters (EOP) were corrected by downloading tables of the measured EOP for the time of each observation. We also corrected ionospheric and tropospheric delays - these are discussed in section 3.3 below.

The phase calibrator sources used in the observations are compact extragalactic core-jet sources (e.g. Mutel et al. (1998)). We made self-calibrated images of the calibrators (SNR 5000:1) which we used to recompute the fringe solution. In principle, the core position of a calibrator could shift with frequency. However, Fomalont et al. (2011) observed four ICRF calibrators from $8-43 \mathrm{GHz}$ and found the core positions are coincident to within 0.02 mas. Hence, we have assumed that the core centroids of the calibrators have a negligible shift from 8-15 GHz.

For the Algol observations, we also corrected for source motion within the observing period. Since the radio emission in Algol is associated with the $\mathrm{K}$ sub-giant (Lestrade et al. 1993), it may move nearly 2 mas during a 12-hour observation, especially if the observation is centered near one of the eclipses. Images made without correcting for this motion would result in a smeared-out source along the direction of motion. Using the already well-determined orbital elements of the inner binary in Algol, we introduced a time-varying phase-tracking correction to the visibilities (using AIPS task CLCOR) equal to the $\mathrm{K}$ star's position offset in the inner binary orbit.

In order to determine radio centroid positions, we made Stokes I images using target visibilities that had been corrected from linearly interpolated delay-rate and phase solutions of calibrator scans bracketing each target scan. We then self-calibrated these images in order to improve image fidelity and better discern the source structure. If the source was unresolved, we fit a Gaussian to the centroid of the emission (AIPS task JMFIT) and used the Gaussian FWHM/2 as the position uncertainty estimate. For epochs with a resolved double structure we found the centroid of each radio lobe and used the midpoint between the lobes as the radio position for that epoch. In both cases, the position uncertainties were increased if the calculated position uncertainty due to tropospheric and ionospheric delays (section 3.3) exceeded the imagebased estimate.

\subsection{Parameter Fitting}

We performed a global least-squares minimization of the orbital and astrometric parameters designated 'variable' in Tables 3 and 4 using our VLBI data as well as radial velocity data (Duemmler \& Aarum 2001; Hill et al. 1971; Hill et al. 1993; Güdel et al. 1999) and differential positions from the Fourth Catalog of Interferometric Positions of Binary Stars (Hartkopf et al. 2010). We began with a coarse grid search, varying each parameter by small steps over a reasonable range of values and finding the minimum $\chi^{2}$-value in the resulting multi-dimensional parameter space. We used the NelderMead simplex (a.k.a. 'amoeba') algorithm (Press et al. 1992) to refine the best-fit parameter values from the coarse grid search. This minimization scheme finds the direction of steepest descent without needing to calculate partial derivatives, which is especially useful when solving transcendental orbital equations. It also uses an adaptive stepsize in order to converge to within a very small tolerance of the minimum chi-square solution in the parameter space.

\subsection{Astrometric accuracy}

An ideal interferometer with projected baseline length $B$ has a positional accuracy given by (Thompson et al. 1986)

$$
\sigma=\frac{1}{2 \pi} \frac{1}{S N R} \frac{\lambda}{B},
$$

where $S N R$ is the signal-to-noise ratio of the target source and $\lambda$ the observing wavelength. This ideal estimate, of order several $\mu$ as for centimeter-wavelength VLBI, is almost never realized in practice since instrumental and intervening atmospheric effects degrade the phase of the incoming signals.

Phase-referenced astrometric observations using modern VLBI arrays typically have very small antenna position and timing uncertainties. Neither of these uncertainties contribute significantly to the astrometric accuracy so long as the switching time between calibrator and source is smaller than the coherence time. The two most important sources of position uncertainty result from path length fluctuations in the troposphere and the ionosphere (e.g. Fomalont et al. 1995, Ros \& Reid 2005: Pradel et al. 2006). A good phase-referencing scheme (i.e. with a short enough cycle time) should compensate for these delays, but for sub-mas astrometry they can still be a source of error and should be addressed.

The daytime ionospheric delay is dispersive $\left(\tau \propto \lambda^{2}\right)$ and is highly dependent on time of day. At $10 \mathrm{GHz}$, the daytime zenith ionospheric delay is quite large, typically $1 \mathrm{~ns}$, corresponding to an excess path length $30 \mathrm{~cm}$. Fortunately, since 1998 there have been several global databases of ionospheric delay maps available for online download. These maps are generated every two hours and are derived from observed GPS delays (Ros et al. 2000). We have corrected all post-1998 VLBI data for these delays (AIPS task TECOR). Ros et al. (2000) found that the residual ionospheric uncertainty after this correction is less than $\pm 0.15 \mathrm{~ns}(4.5 \mathrm{~cm})$ at $8.4 \mathrm{GHz}$ on global baselines.

The tropospheric delay is non-dispersive, and consists of a dry component well predicted by the local atmospheric pressure, and a highly variable wet component whose value can exceed $10 \mathrm{~cm}$ excess path length. Both GPS delay maps and water vapor radiometers have been used to measure and correct for the wet component (e.g. Snajdrova et al. 2006), reducing the uncertainty to about 
TABLE 1

VLBI OBSERVING LOG

\begin{tabular}{|c|c|c|c|c|c|c|}
\hline Exp. Code & Nr. epochs & Dates & Freq. (GHz) & Cal. Source & Array & Ref. \\
\hline \multicolumn{7}{|c|}{ Algol } \\
\hline BM044 & 1 & 1995.309 & 8.4 & $0313+412$ & VLBA + Y 27 & Mutel et al. (1998) \\
\hline BM074 & 3 & $1997.303-1997.308$ & 8.4 & $0313+412$ & $\mathrm{VLBA}+\mathrm{Y} 27+\mathrm{EB}$ & \\
\hline BM109 & 3 & $1999.042-1999.058$ & 8.4 & $0313+412$ & $\mathrm{VLBA}+\mathrm{Y} 27+\mathrm{EB}$ & \\
\hline BM267 & 6 & $2008.265-2008.628$ & 15.4 & $0313+412$ & $\mathrm{VLBA}+\mathrm{GBT}+\mathrm{EB}$ & Peterson et al. (2010) \\
\hline \multicolumn{7}{|c|}{ UX Arietis } \\
\hline- & 9 & $1983.568-1994.405$ & 8.4 & $0326+277$ & (a) & Lestrade et al. (1999) \\
\hline BB032 & 1 & 1994.833 & 8.4 & $0326+277$ & VLBÁ & Beasley \& Gudel (2000) \\
\hline BB049 & 6 & $1995.875-1995.885$ & 8.4 & $0326+277$ & VLBA & \\
\hline BG097 & 1 & 2001.128 & 8.4 & $0326+277$ & $\mathrm{VLBA}+\mathrm{EB}$ & \\
\hline BM140 & 4 & $2001.728-2001.739$ & 8.4 & $0326+277$ & $\mathrm{VLBA}+\mathrm{EB}$ & Massi et al. (2002) \\
\hline BP157 & 4 & $2009.638-2009.797$ & 15.4 & NRAO140 & $\mathrm{VLBA}+\mathrm{GBT}+\mathrm{EB}$ & This paper \\
\hline
\end{tabular}

a VLBI arrays varied with epoch, see Lestrade et al. (1999)

$3 \mathrm{~cm}$. However, although all VLBA sites measure the local atmospheric pressure and hence the dry component accurately, they are not equipped with water vapor radiometers, so there is no on-site measurement of wet component delay. Rather, the correlator applies a seasonally-based average value, which can be in error by several $\mathrm{cm}$ on a given day, especially for humid sites such as St. Croix.

For phase-referenced observations on a single baseline, these path delay uncertainties result in a position uncertainty given by (cf. Reid et al. 1999)

$$
\delta \theta=\left(\frac{c \delta \tau_{0}}{\lambda}\right) \tan (Z) \sec (Z) \cdot \delta Z \cdot \Theta,
$$

where $Z$ is the mean zenith angle, $\delta Z$ is the zenith angle difference between calibrator and target, $\delta \tau_{0}$ is the zenith path length uncertainty, and $\Theta$ is the baseline angular resolution.

In Figure 1 we show a representative plot of the positional uncertainty expected from ionospheric and tropospheric delay uncertainties as a function of frequency for a global VLBI array using the nodding technique at mean zenith angle $70^{\circ}$ and target-calibrator angular separations $1^{\circ}$ and $4^{\circ}$. We have also assumed a tropospheric uncertainty $\pm 0.1 \mathrm{~ns}(3 \mathrm{~cm})$, and mean daytime ionospheric uncertainty of $\pm 0.1 \mathrm{~ns}(3 \mathrm{~cm})$. Nighttime ionospheric delay fluctuations are much smaller ( $\sim 10 \%$ daytime values) and are unimportant at frequencies above a few $\mathrm{GHz}$.

It is clear that at $15 \mathrm{GHz}$, the tropospheric contribution dominates, resulting in position uncertainties 0.12 mas at $1^{\circ}$ calibrator-target separation and 0.5 mas at $4^{\circ}$ separation. At $8.4 \mathrm{GHz}$, the daytime ionospheric and the tropospheric contributions are nearly equal, resulting in a total uncertainty 0.18 mas at $1^{\circ}$ separation and 0.68 mas uncertainty at $4^{\circ}$ separation. Unless given otherwise, we use these uncertainties as formal uncertainties for all VLBI measurements in Table 1

\section{RESULTS}

Table 2 lists radio centroid positions for both sources determined at all VLBI observing epochs. The global least-squares astrometric solutions computed from these

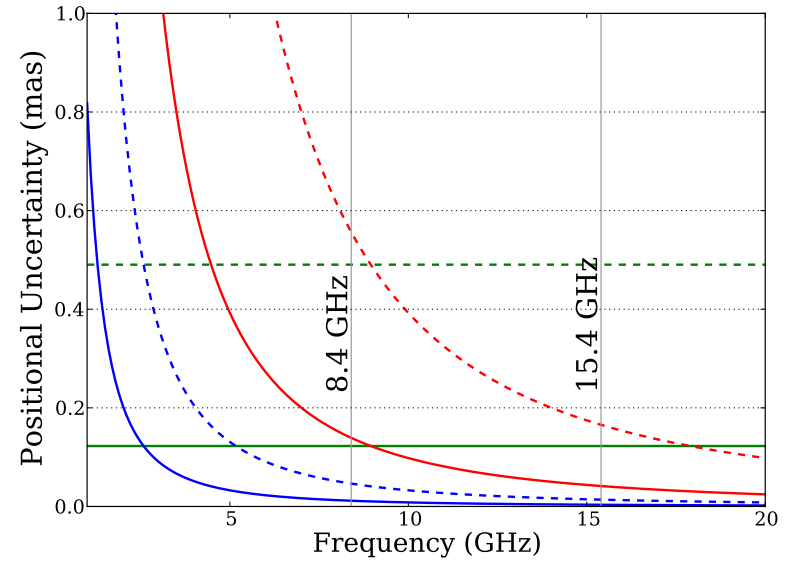

FIG. 1.- Position uncertainty vs. frequency for baseline length $10^{4} \mathrm{~km}, 70^{\circ}$ zenith angle, tropospheric delay uncertainty $\pm 0.1 \mathrm{~ns}$ $(3 \mathrm{~cm})$ and daytime ionospheric delay uncertainty $\pm 0.1 \mathrm{~ns}(3 \mathrm{~cm})$. The solid and dashed lines show position uncertainties for targetcalibrator separations of $1^{\circ}$ and $4^{\circ}$, respectively. Position uncertainties due to tropospheric delay uncertainty are shown by the green lines, the daytime ionosphere contribution is shown by red lines, and the nighttime ionosphere contribution is blue. The $15.4 \mathrm{GHz}$ position uncertainties are dominated by the troposphere, while at $8.4 \mathrm{GHz}$, the daytime ionospheric contribution is comparable to the tropospheric contribution.

positions are listed in Tables 3 and 4 . The corresponding differences between observed positions at each epoch and those calculated using the global solution are given in Table 5. where the column labeled $\sigma$ is the number of standard deviations between the observed and model positions. For both solutions, the agreement is excellent, with only a few epochs marginally above $2 \sigma$.

\subsection{Algol}

\subsubsection{Outer orbit}

Figure 3 shows the position of the radio-loud secondary (Algol B) on the sky, after correction for proper motion and parallax. The model trajectory is shown as gray line, while the observed and predicted positions at each observing epoch are shown as blue errorbars and red $\times$ 's, respectively. Our outer orbital solution agrees very well with Zavala et al. (2010), including the orientation of the 
TABLE 2

OBSERVED RADIO CENTROID POSITIONS

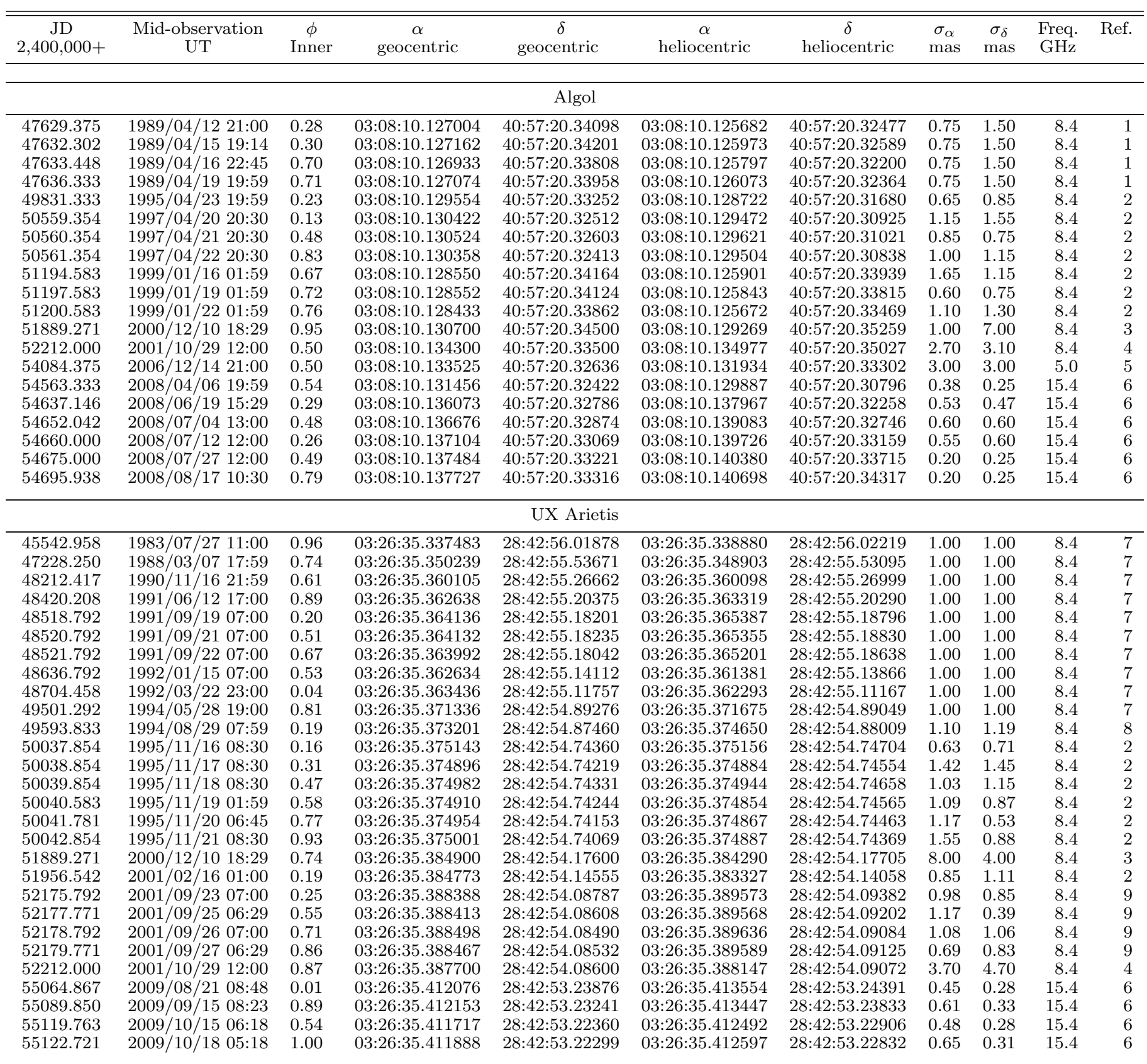

Note. - (a) Heliocentric positions were calculated using the parallax given in Tables 3 and 4 (b) Coordinates were measured with respect to primary phase calibrators: 0313+412 (03:13:01.962129, +41:20:01.18353), 0326+277 (03:29:57.669425, +27:56:15.49901), NRAO140 (03:36:30.107609, +32:18:29.34226) (Ma et al. 1998 Fey et al. 2004). (c) Previously published star coordinates have been corrected for different assumed calibrator positions if needed. (d) References: 1. Lestrade et al. (1993), 2. Positions from VLBA archival data, 3. Boboltz et al. (2003), 4. Fey et al. (2006), 5. Csizmadia et al. (2009), 6. This paper, 7. Lestrade et al. (1984 1999) and unpublished positions, 8. Beasley \& Gudel (2000), position calculated trom VLBA archival data 9. Franciosinı et al. (1999), positions calculated from VLBA archival data. 
TABLE 3

Algol orbital Element Solutions

\begin{tabular}{|c|c|c|c|c|}
\hline Parameter & Symbol & Value & unit & Param.type \\
\hline Parallax & $\Pi$ & $34.7 \pm 0.6$ & mas & Variable \\
\hline Primary mass & $m_{A}$ & 3.70 & $M_{\odot}$ & Fixed \\
\hline Secondary mass & $m_{B}$ & 0.79 & $M_{\odot}$ & Fixed \\
\hline Tertiary mass & $m_{C}$ & $1.51 \pm 0.02$ & $M_{\odot}$ & Calculated \\
\hline R.A. proper motion & $\mu_{\alpha \cos \delta}$ & $2.70 \pm 0.07$ & $\operatorname{mas} \mathrm{yr}^{-1}$ & Variable \\
\hline Dec. proper motion & $\mu_{\delta}$ & $-0.80 \pm 0.09$ & $\operatorname{mas} \mathrm{yr}^{-1}$ & Variable \\
\hline Fiducial $^{\mathrm{a}}$ R.A. & $\alpha_{0}$ & $03: 08: 10.132409 \pm 0.7$ & mas & Variable \\
\hline Fiducial $^{\mathrm{a}}$ Declination & $\delta_{0}$ & $40: 57: 20.3353 \pm 0.6$ & mas & Variable \\
\hline Fiducial epoch & $J D_{0}$ & 2452212.02 & JD & Fixed \\
\hline & & 2001.82553 & year & \\
\hline \multicolumn{5}{|c|}{ Inner binary } \\
\hline Period & $P_{1}$ & 2.867329 & days & Fixed \\
\hline Eccentricity & $e_{1}$ & 0.000 & & Fixed \\
\hline Inclination & $i_{1}$ & $99 \pm 17$ & deg & Variable \\
\hline Longitude of ascending node & $\Omega_{1}$ & $48 \pm 20$ & deg & Variable \\
\hline Lonigtude of periastron & $\omega_{1}$ & 270 & deg & Fixed \\
\hline Semi-major axis & $a_{1}$ & 2.26 & mas & Fixed \\
\hline \multirow{2}{*}{ Time periastron } & $T_{1}$ & 2441773.49 & JD & Fixed \\
\hline & & 1973.24638 & year & \\
\hline \multicolumn{5}{|c|}{ Outer binary } \\
\hline Period & $P_{2}$ & $679.5 \pm 0.3$ & days & Variable \\
\hline Eccentricity & $e_{2}$ & $0.16+0.02$ & years & Variable \\
\hline Inclination & $i_{2}$ & $85.5 \pm 1.4$ & $\operatorname{deg}$ & Variable \\
\hline Longitude of ascending node & $\Omega_{2}$ & $130.7 \pm 3.5$ & deg & Variable \\
\hline Longitude of periastron & $\omega_{2}$ & $312.0 \pm 1.4$ & deg & Variable \\
\hline Semi-major axis & $a_{2}$ & $95.4 \pm 0.5$ & mas & Variable \\
\hline \multirow[t]{2}{*}{ Time periastron } & $T_{2}$ & $2446930.0 \pm 3.2$ & JD & Variable \\
\hline & & 1987.36551 & year & \\
\hline
\end{tabular}

a Center of mass of triple system at fiducial epoch 
TABLE 4

UX ARIETIS ORBITAL ELEMENT SOLUTIONS

\begin{tabular}{|c|c|c|c|c|}
\hline Parameter & Symbol & Value & unit & Param. type \\
\hline $\begin{array}{l}\text { Parallax } \\
\text { Primary mass } \\
\text { Secondary mass } \\
\text { Tertiary mass } \\
\text { R.A. proper motion } \\
\text { Dec. proper motion } \\
\text { Fiducial }{ }^{\mathrm{a}} \text { R.A. } \\
\text { Fiducial }^{\mathrm{a}} \text { Declination } \\
\text { Fiducial epoch }^{\text {Fiducial epo }}\end{array}$ & $\begin{array}{r}\Pi \\
m_{A} \\
m_{B} \\
m_{C} \\
\mu_{\alpha \cos \delta} \\
\mu_{\delta} \\
\alpha_{0} \\
\delta_{0} \\
J D_{0}\end{array}$ & $\begin{array}{r}19.90 \\
1.10 \\
0.95 \\
0.75 \pm 0.01 \\
44.96 \pm 0.13 \\
-102.33 \pm 0.09 \\
03: 26: 35.38386 \pm 1.2 \\
28: 42: 54.2755 \pm 0.8 \\
2451544.5 \\
2000.0\end{array}$ & $\begin{array}{l}\text { mas } \\
M_{\odot} \\
M_{\odot} \\
M_{\odot} \\
\text { mas } \mathrm{yr}^{-1} \\
\text { mas } \mathrm{yr}^{-1} \\
\text { mas } \\
\text { mas } \\
\text { JD } \\
\text { year }\end{array}$ & $\begin{array}{l}\text { Fixed } \\
\text { Fixed } \\
\text { Fixed } \\
\text { Calculated } \\
\text { Variable } \\
\text { Variable } \\
\text { Variable } \\
\text { Variable } \\
\text { Fixed }\end{array}$ \\
\hline \multicolumn{5}{|c|}{ Inner binary } \\
\hline $\begin{array}{l}\text { Period } \\
\text { Eccentricity } \\
\text { Inclination } \\
\text { Longitude of ascending node } \\
\text { Longitude of periastron } \\
\text { Semi-major axis } \\
\text { Time periastron }\end{array}$ & $\begin{array}{r}P_{1} \\
e_{1} \\
i_{1} \\
\Omega_{1} \\
\omega_{1} \\
a_{1} \\
T_{1}\end{array}$ & $\begin{array}{r}6.4378553 \\
0.000 \\
59.2 \\
82 \pm 34 \\
180.0 \\
1.71 \\
2450642.00075 \\
1997.52705\end{array}$ & $\begin{array}{l}\text { days } \\
\text { deg } \\
\text { deg } \\
\text { deg } \\
\text { mas } \\
\text { JD } \\
\text { year }\end{array}$ & $\begin{array}{l}\text { Fixed } \\
\text { Fixed } \\
\text { Fixed } \\
\text { Variable } \\
\text { Fixed } \\
\text { Fixed } \\
\text { Fixed }\end{array}$ \\
\hline \multicolumn{5}{|c|}{ Outer binary } \\
\hline $\begin{array}{l}\text { Period } \\
\text { Eccentricity } \\
\text { Inclination } \\
\text { Longitude of ascending node } \\
\text { Longitude of periastron } \\
\text { Semi-major axis } \\
\text { Time periastron }\end{array}$ & $\begin{array}{r}P_{2} \\
e_{2} \\
i_{2} \\
\Omega_{2} \\
\omega_{2} \\
a_{2} \\
T_{2}\end{array}$ & $\begin{array}{r}40548.6 \pm 70.2 \\
111.02 \\
0.77 \pm 0.01 \\
93.3 \pm 0.6 \\
58.9 \pm 0.5 \\
274.9 \pm 0.8 \\
648.0 \pm 0.7 \\
2451664.9 \pm 34.3 \\
2000.32964\end{array}$ & $\begin{array}{l}\text { deg } \\
\text { deg } \\
\text { deg } \\
\text { mas } \\
\text { JD } \\
\text { year }\end{array}$ & $\begin{array}{l}\text { Variable } \\
\text { Variable } \\
\text { Variable } \\
\text { Variable } \\
\text { Variable } \\
\text { Variable } \\
\text { Variable }\end{array}$ \\
\hline
\end{tabular}

${ }^{a}$ Center of mass of triple system at fiducial epoch

Algol C orbit, which differed from earlier determinations (see Zavala et al. for discussion). We note that the derived mass of the tertiary component $\left(1.57 \pm 0.01 M_{\odot}\right)$, although determined to high accuracy, depends on the masses of A and B, which we have assumed are exactly known.

We can use the outer orbit solution to compute the orbit of Algol C relative to the AB center of mass and compare with optical observations. The model AB-C orbit is shown in Figure 2, green line) along with optical observations of Algol C tabulated in the Fourth Catalog of Interferometric Measurements of Binary Stars (FCIMBS) (Hartkopf et al. 2010). The observed positions are in good agreement with the model orbit and predicted positions at each observing epoch.

\subsubsection{Inner orbit}

Figure 4 shows the orbit of Algol B in the inner binary center of mass frame, along with the mean observed (blue errorbars) and model (red $\times$ 's) positions at the six observing epochs observed at $15 \mathrm{GHz}$ (experiment BM267). We have not displayed the $8 \mathrm{GHz}$ positions, since their position uncertainty is twice as large as the $15 \mathrm{GHz}$ points and provide little additional constraint of the inner binary orbit. The orientation of the inner binary, $\Omega=48^{\circ} \pm 20^{\circ}$, agrees with the earlier VLBI result of Lestrade et al. (1993) $\left(52^{\circ} \pm 5^{\circ}\right)$ and the more recent optical determination on Zavala et al. (2010) $\left(47.4^{\circ} \pm 5.2^{\circ}\right)$. We also find $i=99^{\circ} \pm 17^{\circ}$, confirming the conclusion of Zavala et al. (2010), who found that the inner orbit is retrograde (i.e. $i>90^{\circ}$, contrary to the previous deter-

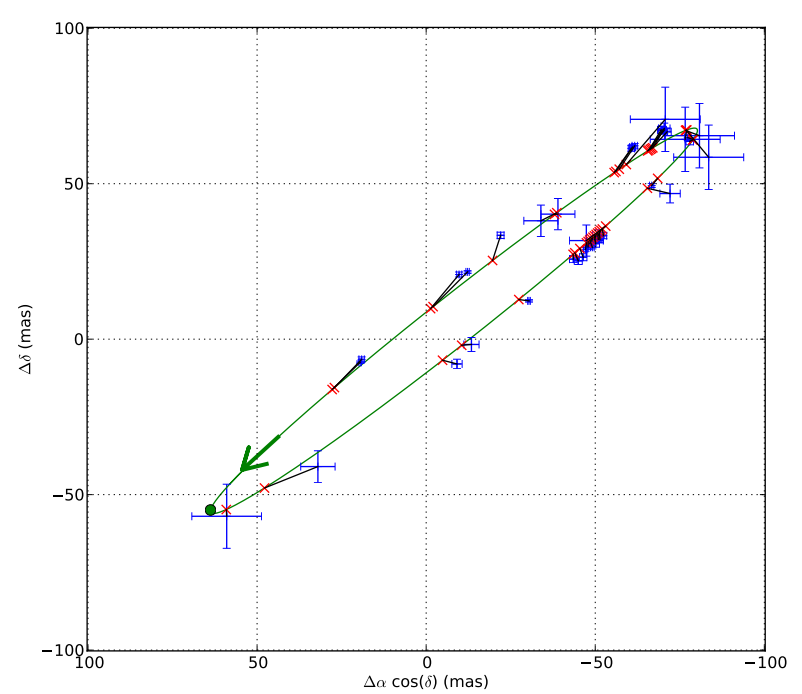

Fig. 2.- Algol C (tertiary) orbit with respect to the $\mathrm{AB}$ center of mass determined from VLBI global astrometric solution (green line) along with speckle interferometer observations listed in the Fourth Catalog of Interferometric Measurements of Binary Stars (Hartkopf et al. 2010) (blue errorbars) and model positions for the corresponding dates (red $\times$ 's). The green arrow is positioned with its tail at the point of periastron, and shows the direction of orbit. The green circle is at the ascending nodal point. 


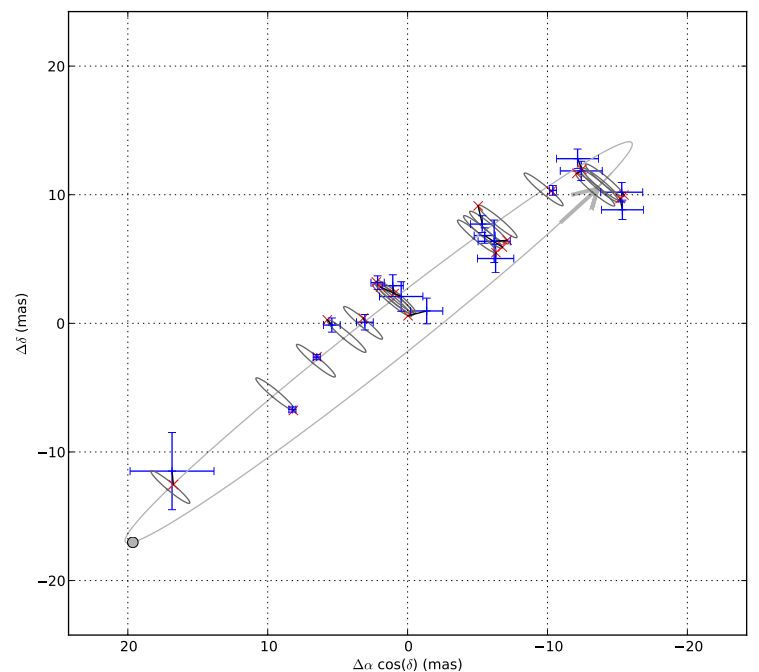

Fig. 3.- Orbital path of the Algol inner binary center of mass (light gray line), with VLBI observations (blue errorbars) and model positions of the radio-loud star (Algol B, red X's). The model orbit of Algol B is shown at the time of each observation (dark gray ovals) for reference. The gray arrow shows the orbital direction for the $\mathrm{AB}$ center of mass, and the gray circle is the ascending node.

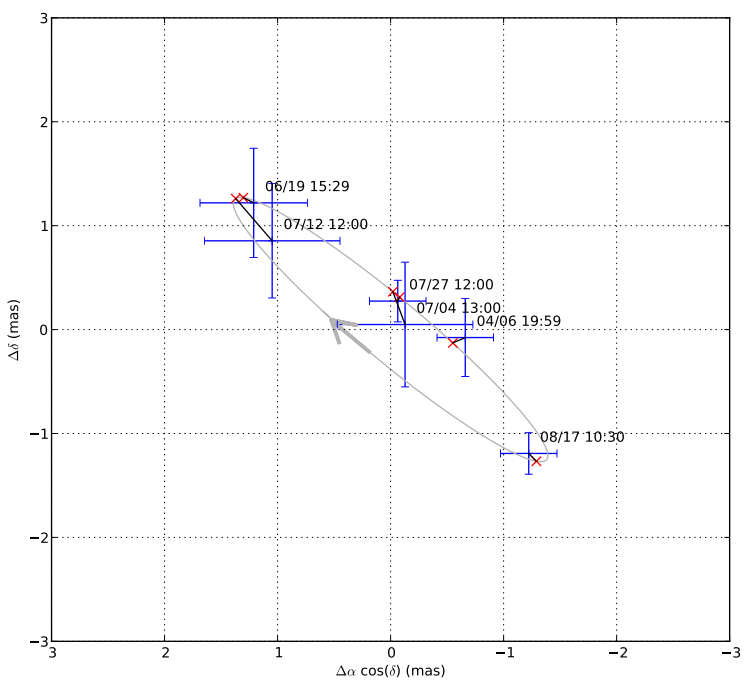

FiG. 4.- Algol inner binary system, showing model orbit (gray line), and observed (blue errorbars) and predicted (red $\times$ 's) radio centroids at six epochs between 2008.27 - 2008.63. The gray arrow shows the direction of orbital travel. The ascending node of the orbit lies toward the upper-left in the figure.

mination of Csizmadia et al. (2009).

\subsubsection{Algol's radio morphology: Co-rotating coronal loop centered on KIV star}

Algol's radio morphology consists of a double-lobed structure oriented normal to the inner orbital plane during quiescent states, and a crescent or loop structure during active periods (see Peterson et al. 2010, and supplementary materials). The registration between radio and
TABLE 5

VLBI - MODEL POSITION DIFFERENCES

\begin{tabular}{cccc}
\hline \hline $\begin{array}{c}\text { JD } \\
2,400,000+\end{array}$ & $\begin{array}{c}\Delta \alpha \\
\text { mas }\end{array}$ & $\begin{array}{c}\Delta \delta \\
\text { mas }\end{array}$ & $\begin{array}{c}\sigma \\
\text { mas }\end{array}$ \\
\hline \multicolumn{4}{c}{ Algol } \\
\hline 47629.375 & 0.3 & -0.2 & 0.4 \\
47632.302 & -0.3 & -0.8 & 0.7 \\
47633.448 & 0.2 & 0.9 & 0.6 \\
47636.333 & -0.2 & -0.2 & 0.3 \\
49831.333 & 0.3 & 1.4 & 1.7 \\
50559.354 & 1.5 & 0.7 & 1.4 \\
50560.354 & -0.1 & -0.6 & 0.8 \\
50561.354 & 1.3 & -0.4 & 1.4 \\
51194.583 & -0.9 & 0.1 & 0.6 \\
51197.583 & -1.3 & -0.9 & 2.4 \\
51200.583 & -0.0 & 0.5 & 0.4 \\
54084.375 & -0.1 & -1.1 & 0.4 \\
54563.333 & 0.1 & -0.1 & 0.4 \\
54637.146 & 0.1 & 0.1 & 0.2 \\
54652.042 & 0.1 & 0.3 & 0.6 \\
54660.000 & 0.3 & 0.4 & 0.9 \\
54675.000 & -0.0 & 0.0 & 0.2 \\
54695.938 & -0.1 & -0.1 & 0.5
\end{tabular}

\begin{tabular}{|c|c|c|c|}
\hline \multicolumn{4}{|c|}{ UX Arietis } \\
\hline 45542.958 & 0.9 & -1.5 & 1.7 \\
\hline 47228.250 & -0.8 & -0.2 & 0.8 \\
\hline 48212.417 & 0.7 & 0.9 & 1.1 \\
\hline 48420.208 & 1.1 & 0.8 & 1.4 \\
\hline 48518.792 & $\begin{array}{r}1.1 \\
-0.2\end{array}$ & 0.6 & 0.6 \\
\hline 48520.792 & -1.4 & -0.0 & 1.4 \\
\hline 48521.792 & 0.7 & 2.0 & 2.1 \\
\hline 48636.792 & -1.3 & -0.2 & 1.3 \\
\hline 48704.458 & -1.3 & 0.7 & 1.5 \\
\hline 49501.292 & -0.4 & 0.9 & 0.9 \\
\hline 49593.833 & -0.6 & -0.7 & 0.8 \\
\hline 50037.854 & -0.5 & -1.2 & 1.8 \\
\hline 50038.854 & 1.8 & -0.3 & 1.3 \\
\hline 50039.854 & -0.1 & -1.5 & 1.3 \\
\hline 50040.583 & 0.7 & -0.7 & 1.0 \\
\hline 50041.781 & 0.6 & 0.1 & 0.5 \\
\hline 50042.854 & 0.4 & 0.4 & 0.5 \\
\hline 50039.615 & -1.6 & -1.5 & 1.8 \\
\hline 50040.583 & 0.5 & -0.6 & 1.2 \\
\hline 50041.615 & 0.4 & 0.7 & 1.3 \\
\hline 51956.542 & -1.2 & -1.2 & 1.7 \\
\hline 52175.792 & 1.3 & -1.4 & 2.0 \\
\hline 52177.771 & -0.2 & 0.2 & 0.6 \\
\hline 52178.792 & -0.9 & 1.4 & 1.6 \\
\hline 52179.771 & 0.1 & 0.7 & 0.9 \\
\hline 55064.867 & 0.1 & 0.1 & 0.4 \\
\hline 55089.850 & -0.6 & 0.4 & 1.5 \\
\hline 55119.763 & 0.6 & -0.1 & 1.3 \\
\hline 55122.721 & -0.6 & -0.3 & 1.4 \\
\hline
\end{tabular}

optical maps cannot be made directly, since the optical position uncertainty ellipse $(5 \times 8$ mas, Perryman et al. (1997)) is larger than the inner binary orbit. However, our $15.4 \mathrm{GHz}$ observations show that the radio centroid mirrors the predicted motion of the KIV secondary over six epochs to an uncertainty \pm 0.5 mas.

It is possible that the radio centroid is systematically displaced from the $\mathrm{K}$ star and is following its trajectory precisely, but this seems unphysical. Occam's razor leads us to conclude that the correct registration is that the radio centroid is coincident with the $\mathrm{K}$ star center. In that case, the lobe structure is straddling the active KIV secondary, since the lobe separation ( 1.0 mas) is the same as the K star's angular diameter (1.1 mas). As discussed by Peterson et al. (2010, and online supplement), 
the radio morphology and registration is consistent with a co-rotating, plasma-filled coronal loop structure emitting synchrotron radiation. The loop is oriented in the direction of the primary, which may imply magnetic interaction with the primary's accretion disk (e.g., Retter et al. 2005).

X-ray observations (Chung et al. 2004) show that the centroid of the quiescent emission orbits with a semimajor axis $15 \%$ smaller than the K star - i.e. it is offset toward the center of mass of the inner binary. The radio positions in Figure 4 appear likewise shifted inward of the $\mathrm{K}$ star orbit. Our global astrometric solution found that the semi-major axis of the centroid of the radio emission is shifted inward by $0.2 \pm 0.8$ mas. It is worth noting that the shift in the radio centroid is flux-dependent, moving inward toward the top of the co-rotating loop when the high-flux events fill the loop.

\subsection{UX Arietis}

Unlike Algol, the orbital elements of a third component in the UX Arietis system were not previously known, although the presence of a third component is not unexpected - companions in close binaries are quite common. Tokovinin et al. (2006) found that $63 \%$ of spectroscopic binaries (SB) that they surveyed had at least a tertiary companion. For SB's with a period less than 3 days, the fraction with companions is $96 \%$, suggesting that the shorter period systems exchanged angular momentum with their companions, shortening their orbital periods.

In the UX Arietis system, a third component had been inferred by detection of non-linear proper motion (e.g., Boboltz et al. 2003) and spectral lines of a possible third component (Aarum Ulvås \& Engvold 2003a). However, the putative third component's orbit was poorly determined. The only published orbital solution consisted of

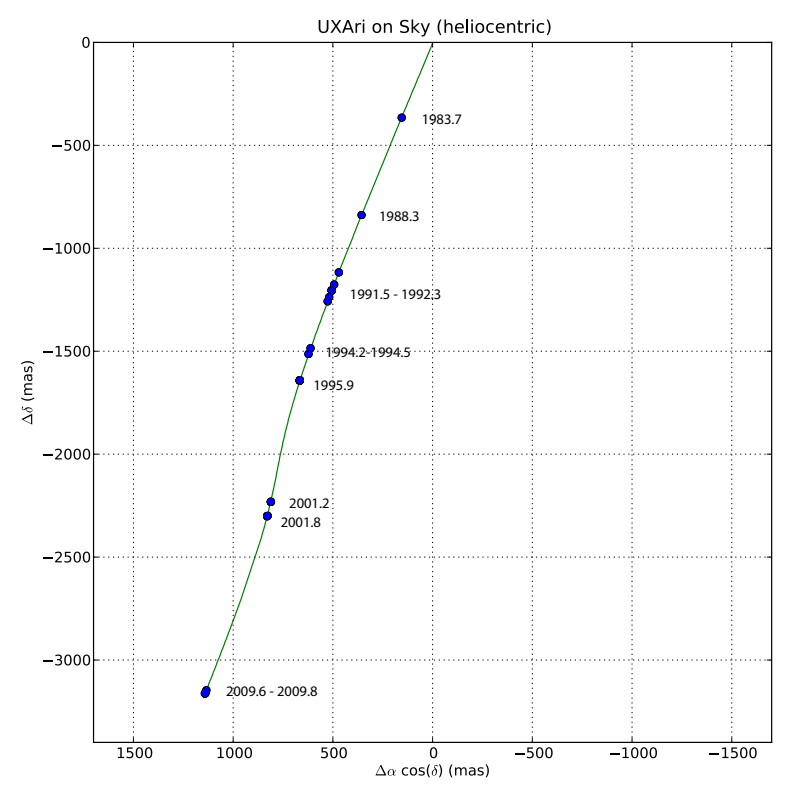

FIG. 5.- UX Arietis radio position projected on the sky plane since 1980.0. The undulating trajectory results from proper motion combined with reflex motion in the $\mathrm{AB}-\mathrm{C}$ outer binary. two quite different models: a $10.7 \mathrm{yr}$ circular orbit and a 21.5 yr highly eccentric orbit (Duemmler \& Aarum 2001). Therefore, it was necessary to undertake a comprehensive parameter search, constrained by the VLBI positions, third component radial velocity data (Duemmler \& Aarum 2001; Aarum Ulvås \& Engvold 2003a; Glazunova et al. 2008), and optical interferometric observations in the FCIMBS (Hartkopf et al. 2010). The search was complicated by the relatively high proper motion of UX Arietis $\left(\mu \sim 100\right.$ mas $\left.\mathrm{yr}^{-1}\right)$, which magnifies a small fractional proper motion uncertainty over decades into a large aggregate position shift.

\subsubsection{Outer orbit}

Figure 5 shows the radio position of UX Arietis on the sky plane, from epoch 1980.0 (origin) to 2009.8. The blue circles are observed positions relative to 1985.0. The undulating shape of the trajectory, previously interpreted as an acceleration (Lestrade et al. 1999, Boboltz et al. 2003 Fey et al. 2006)) results from the reflex motion of the inner binary in a 111-yr period outer orbit. The position of the radio component in the frame of the outer binary center of mass in shown in Figure 6. The gray line is the predicted position of the inner binary center of mass, with blue errorbars indicating observed positions and red X's the model positions after correction for proper motion and parallax.

We next compare the model solution with FCIMBS interferometer observations. Figure 7 shows the model position of the tertiary component with respect to the inner binary center of mass (green line). The red and corresponding blue symbols are the model and observed positions of the tertiary component as listed in the FCIMBS. Note that for all FCIMBS observations prior to 2002, we have applied a $180^{\circ}$ correction to the catalogued position angle. Finally, Figure 8 shows the predicted radial velocity of the inner binary center of mass (gray line) and ter-

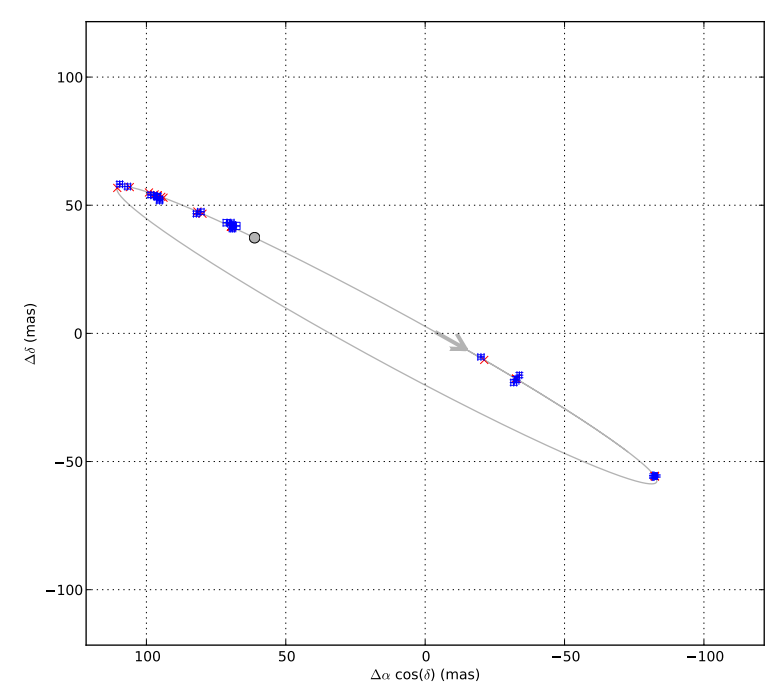

FIG. 6.- Position of UX Arietis primary relative to triple system center of mass, showing model (gray line), and predicted (red $\times$ 's) and observed (blue +'s) positions. The arrow shows the orbital direction and is positioned with its tail at the point of periastron. The gray circle shows the ascending nodal point. 


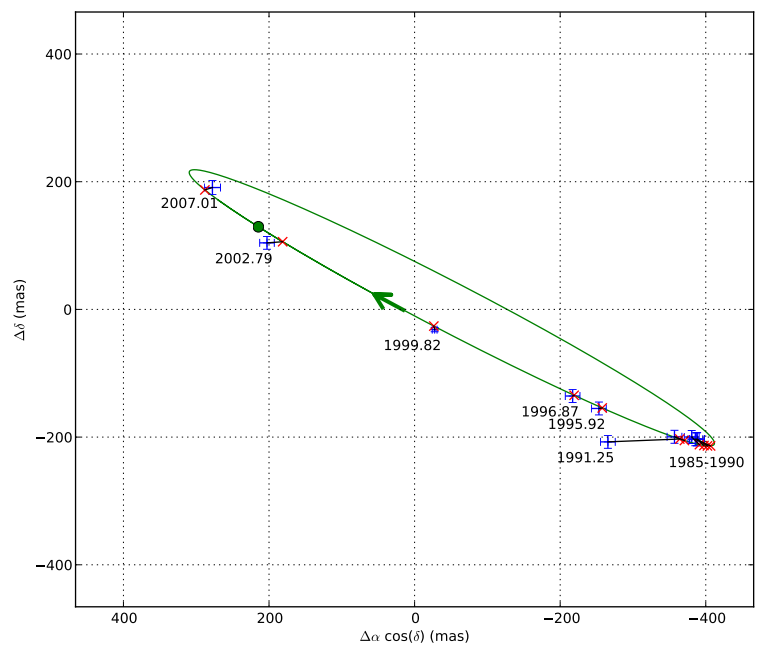

FIG. 7.- UX Arietis C (tertiary) orbit with respect to the AB center of mass determined from VLBI global orbit solution (green line), along with speckle interferometer observations listed in the Fourth Catalog of Interferometric Measurements of Binary Stars (Hartkopf et al. 2010) (blue errorbars) and model positions for the corresponding dates (red $\times$ 's). The green arrow is positioned with its tail at the point of periastron, and shows the direction of orbit. The green circle is at the ascending nodal point.

tiary component (green line), along with corresponding radial velocity observations (Duemmler \& Aarum 2001; Massarotti et al. 2008; Glazunova et al. 2008). In general, the agreement with all three datasets is very good, although the outlier at epoch 1991.25 from the HIPPARCOS catalog (Perryman et al. 1997) is not consistent with the orbit solution.

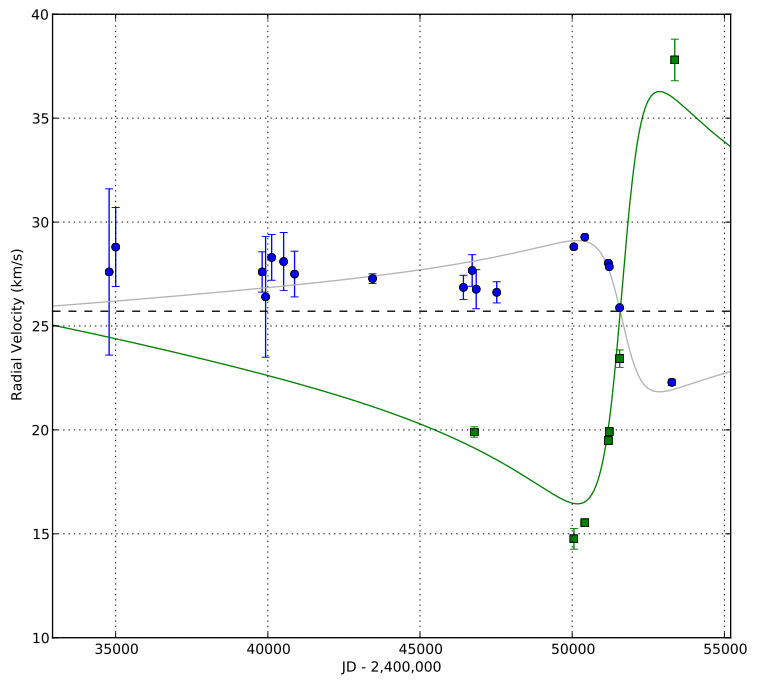

FIG. 8. - UX Arietis outer orbit radial velocities (Duemmler \& Aarum 2001. Massarotti et al. 2008 Glazunova et al. 2008). Model curves based on the outer orbit solution are shown for the inner binary center of mass (gray line) and tertiary (green line), along with observed velocities for the tertiary (green squares) and $\mathrm{AB}$ center of mass (blue circles).

\subsubsection{Inner orbit}

Since both inner binary components of UX Arietis exhibit chromospheric activity (Aarum Ulvås \& Engvold 2003a), it is unclear whether the radio emission originates from a single component, both components or perhaps an accretion region between the two stars. The orbital elements are well-characterized by spectroscopic observations except for the orientation $(\Omega)$ and the inclination ( $i \sim 60^{\circ}$, Duemmler \& Aarum 2001), which is degenerate about $90^{\circ}$ reflection with respect to radial velocity curves. Hence we fixed the other orbital parameters and, as with Algol, used only the $15 \mathrm{GHz}$ observations to solve for for $\Omega$ and $i$.

We confirm that the K0IV primary is the source of the radio emission in UX Arietis by testing the quality of the fit assuming the alternatives: that the emission is either from the secondary, or from a location between the two components. The alternative assumptions both yielded unacceptable fits $\left(\chi_{\nu}^{2}=7.6\right.$ and $\chi_{\nu}^{2}=2.8$, respectively), while the solution for radio emission from the primary was very good, yielding a good fit at the $99.7 \%$ confidence level.

Figure 9 shows the best-fit orbital solution overlaid with contour maps of the radio emission at each epoch. We obtain a tentative solution for $\Omega\left(82^{\circ} \pm 34^{\circ}\right)$. Our data cannot constrain the solution in $i$, as can be seen by the fact that the timing of our observations were all very close to the nodal points in the inner binary orbit. Fixing the inclination at 59.2 ${ }^{\circ}$ (Duemmler \& Aarum 2001) produces a slightly better fit than the R.V.-degenerate value $\left(i=120.8^{\circ}\right)$, thus we use the former value in our final solution.

\subsubsection{Source structure}

For all four VLBI observations made at $15 \mathrm{GHz}$, the self-calibrated radio images are amorphous, with an overall size comparable to the restoring beam $(\sim 0.4 \times 0.8 \mathrm{mas})$. This contrasts with earlier published images of UX Arietis (Mutel et al. 1985, Beasley \& Güdel 2000, Ros \& Massi 2007) which show structure on an angular scale comparable to the binary separation (1.7 mas). However, the previous observations were at lower frequencies ( $5 \mathrm{GHz}$ and $8.4 \mathrm{GHz}$ ) so it is possible that the interferometer phases were corrupted by uncorrected ionospheric delay fluctuations, especially for observations made prior to 1998, when GPS-based ionospheric corrections were unavailable (cf. section 3.3). Alternatively, there may be frequency-dependent extended structure with a steep spectral index that is not detectable at higher frequencies.

\section{DISCUSSION}

\subsection{Mass and spectral type of UX Arietis tertiary component}

The UX Arietis outer orbit solution can be used to derive the mass of the tertiary component, $m_{c}=0.75 \pm$ $0.01 \mathrm{M}_{\odot}$, where the formal uncertainty is contingent on fixed values for the inner binary masses, as given in Table 4 This mass corresponds to a spectral type K1 main sequence star (Zombeck 1990). Duemmler \& Aarum (2001) estimated the mass of UX Arietis' tertiary com- 

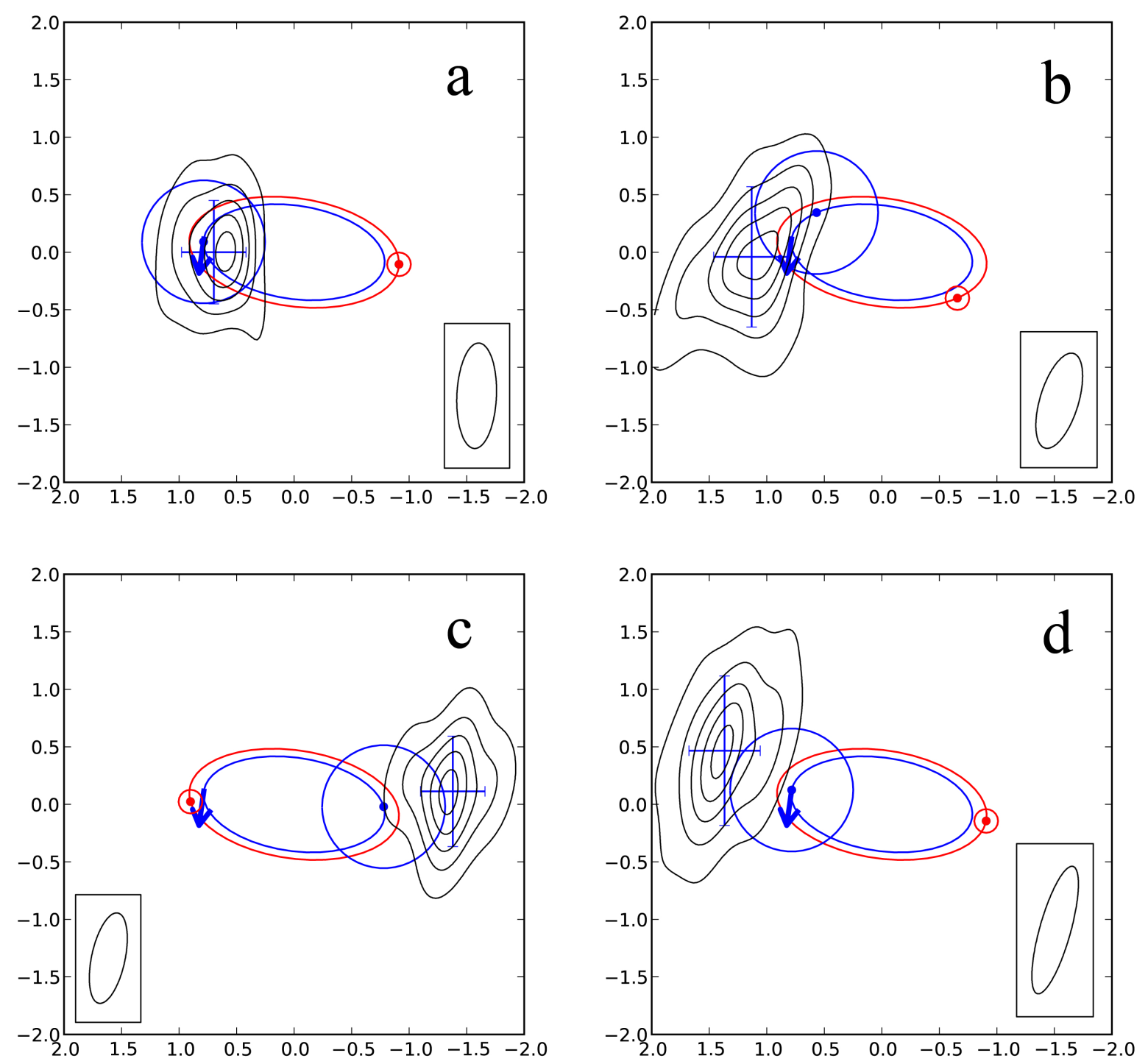

FIG. 9.- UX Arietis inner orbit solution overlaid on radio contour maps at epochs (a) Aug 21, (b) Sep 15, (c) Oct 15, and (d) Oct 18, 2009. Orbits and predicted positions of the KIV primary (blue line, circle) and secondary (red line, circle) are shown. The blue arrow shows the orbital direction and position of the ascending node. The contour levels of the radio images are 10\%, 30\%, 50\%, $70 \%$ and $90 \%$. of peak values $0.7,5.2,3.8$, and $1.4 \mathrm{mJy}_{\text {beam }}{ }^{-1}$ in panels a, b, c, and d respectively. The total flux density at each epoch is (a) $0.9 \mathrm{mJy}$, (b) $9.8 \mathrm{mJy},($ c) $7.5 \mathrm{mJy}$ and (d) $2.8 \mathrm{mJy}$.

ponent between $0.30<m_{3}<0.46 \mathrm{M}_{\odot}$, for their circular and elliptical orbit solutions respectively, although they reject the smaller mass, since it would be an $M$ star too faint to be responsible for the spectral lines ascribed to the tertiary. Aarum Ulvås \& Engvold (2003a) fit the continuum spectrum of the UX Arietis system and found that after correction for the primary and secondary spectra, the derived color index is best-fit to a K5 main-sequence star, although they cast doubt on this identication, since the implied radius ( 0.83 solar radii) is in the range of a K1-type star. Our derived mass agrees with the hotter type, so perhaps starspots on the tertiary are influencing its color index as is suspected with the primary (Aarum Ulvås \& Engvold 2003b).

\subsection{Comparison with polar loop models}

Peterson et al. (2010) recently described a polar loop model for Algol in which the double-lobed radio components are associated with foot points of an active region of meridional magnetic field lines originating at the star's magnetic poles. A similar geometry was already suggested for this system by Franciosini et al. (1999). We now consider whether the single radio component seen in the UX Arietis system can be interpreted within this model.

A large, stable polar spot has been detected on the $\mathrm{K}$ primary of UX Arietis (Vogt \& Hatzes 1991, Elias et al. 1995). These polar spots are a common phenomenon: Starspots on magnetically active cool stars preferentially appear near the poles (e.g. Hatzes et al. 1996: Bruls et al. 1998). This preference for high latitudes may be due to rapid rotation, which leads to the Coriolis force dominat- 
ing over buoyancy forces in the dynamics of magnetic flux tubes. As a consequence, flux tubes in the stellar convection zone migrate nearly parallel to the axis of rotation and thus surface at high latitudes (Schuessler \& Solanki 1992). Hence, magnetically active stars with rapid rotation exhibit magnetic flux eruption at high latitudes and polar starspots.

The VLBI images of UX Arietis indicate that the radio centroid lies in the hemisphere facing away from the $\mathrm{G}$ star. This is consistent with observations of a similar late-type binary, HR1099, in which doppler imagery (Donati et al. 1992) and X-ray monitoring indicate that the active regions lie in the hemisphere facing away from the inactive companion. Audard et al. (2001) suggest that this is because tidal locking of the rotation of the $\mathrm{K}$ star alters the internal dynamo in such a way that strong activity on the hemisphere facing the inactive star is suppressed.

Since the inner binary orbital plane is inclined at $30^{\circ}$ to the observer's line of sight $\left(i \sim 60^{\circ}\right)$, if the magnetic axis is close to perpendicular to the inner binary's orbital plane, only one pole will be visible. The polar loop model posits that the radio emission arises above both polar regions, but in this case, the far side emission will be largely occulted by the $\mathrm{K}$ star. This geometry may explain the absence of two radio centroids.

\section{SUMMARY}

We have analyzed more than 20 years of phasereferenced VLBI observations to determine accurate (sub-mas) positions of the radio centroids from two active close binaries, Algol and UX Arietis, both of which have distant tertiary companions. We used these posi- tions to calculate proper motions and orbital elements of the inner and outer orbits in both stellar systems.

For Algol, we confirm the early result of Lestrade et al. (1993) that the radio centroid closely tracks the motion of the KIV secondary. Furthermore, the radio morphology, which varies from double-lobed at low flux level to crescent-shaped during active periods, is consistent with synchrotron emission from a large, co-rotating meridional loop centered on the K-star. If this correct, it provides a radio-optical frame tie candidate with a precision \pm 0.5 mas. We also refine the proper motion and outer orbit solutions, confirming the recent result of Zavala et al. (2010) that the inner orbit is retrograde.

For UX Arietis, we find an tertiary orbit solution that accounts for previous VLBI observations of an acceleration term in the proper motion fit, as well as radial velocity curves and speckle observations. The dynamical mass, $0.75 \pm 0.01$ solar masses, supports the identification of the tertiary with a $\mathrm{K} 1$ main sequence star, consistent with third-body color index measurements of Aarum Ulvås \& Engvold (2003a). The inner orbit solution favors emission from the active $K$ primary only, in the hemisphere facing away from the $\mathrm{G}$ star. The radio morphology, consisting of a single, partially resolved emission region, may be associated with the persistent polar spot observed using Doppler imaging.

The authors would like to thank Dr. Bob Zavala of the U.S. Naval Observatory Flagstaff Station for his helpful comments and insights, without which our paper would have been much less complete.

\section{REFERENCES}

Aarum Ulvås, V., \& Engvold, O. 2003a, A\&A, 402, 1043 -. 2003b, A\&A, 399, L11

Audard, M., Güdel, M., \& Mewe, R. 2001, A\&A, 365, L318

Bachmann, P. J., \& Hershey, J. L. 1975, The Astronomical Journal, 80, 836

Balega, I. I., Balega, Y. Y., Hofmann, K. H., Malogolovets, E. V., Schertl, D., Shkhagosheva, Z. U., \& Weigelt, G. 2006, Astronomy and Astrophysics, 448, 703

Beasley, A. J., \& Conway, J. E. 1995, A.S.P. Conference 82, Very Long Baseline Interferometry and the VLBA, 82, 328

Beasley, A. J., \& Güdel, M. 2000, Astrophysical Journal, 529, 961

Boboltz, D. A., Fey, A. L., Johnston, K. J., Claussen, M. J., de Vegt, C., Zacharias, N., \& Gaume, R. A. 2003, Astronomical Journal, 126, 484

Bonneau, D. 1979, Astronomy and Astrophysics, 80, L11

Bourda, G., Charlot, P., Porcas, R. W., \& Garrington, S. T. 2010 , Astronomy and Astrophysics, 520

Bower, G. C., Bolatto, A., Ford, E. B., \& Kalas, P. 2009, The Astrophysical Journal, 701, 1922

Bruls, J. H. M. J., Solanki, S. K., \& Schuessler, M. 1998, A\&A, 336, 231

Buccino, A. P., \& Mauas, P. J. D. 2009, Astronomy and Astrophysics, 495, 287

Carlos, R. C., \& Popper, D. M. 1971, Publications of the Astronomical Society of the Pacific, 83

Chung, S. M., Drake, J. J., Kashyap, V. L., Lin, L. W., \& Ratzlaff, P. W. 2004, Astrophysical Journal, 606, 1184

Clark, B. G., Kellermann, K. I., \& Shaffer, D. 1975, The Astrophysical Journal, 198, L123

Csizmadia, S., Borkovits, T., Paragi, Z., brahm, P., Szabados, L., Mosoni, L., Sturmann, L., Sturmann, J., Farrington, C., McAlister, H. A., ten Brummelaar, T. A., Turner, N. H., \& Klagyivik, P. 2009, The Astrophysical Journal, 705, 436
Deller, A. T., Tingay, S. J., Bailes, M., \& Reynolds, J. E. 2009, The Astrophysical Journal, 701, 1243

Donati, J. F., Brown, S. F., Semel, M., Rees, D. E., Dempsey, R. C., Matthews, J. M., Henry, G. W., \& Hall, D. S. 1992, Astronomy and Astrophysics, 265, 682

Duemmler, R., \& Aarum, V. 2001, Astronomy and Astrophysics, 370, 974

Dzib, S., Loinard, L., Mioduszewski, A. J., Boden, A. F., Rodrguez, L. F., \& Torres, R. M. 2010, The Astrophysical Journal, 718, 610

Ekmekci, F. 2010, Publications of the Astronomical Society of Australia, 27, 1

Elias, N. M., Quirrenbach, A., Witzel, A., Naundorf, C. E., Wegner, R., Guinan, E. F., \& McCook, G. P. 1995, Astrophysical Journal, 439, 983

Fey, A. L., Boboltz, D. A., Gaume, R. A., Johnston, K. J., Garrington, S. T., \& Thomasson, P. 2006, Astronomical Journal, 131, 1084

Fey, A. L., Ma, C., Arias, E. F., Charlot, P., Feissel-Vernier, M., Gontier, A. M., Jacobs, C. S., Li, J., \& MacMillan, D. S. 2004, The Astronomical Journal, 127, 3587

Fomalont, E., Diamond, P. J., \& Napier, P. J. 1995, Astronomical Society of the Pacific, 82, 363

Fomalont, E., Johnston, K., Fey, A., Boboltz, D., Oyama, T., \& Honma, M. 2011, AJ, 141, 91

Fomalont, E., Kopeikin, S., Lanyi, G., \& Benson, J. 2009, The Astrophysical Journal, 699, 1395

Franciosini, E., Massi, M., Paredes, J. M., \& Estalella, R. 1999, Astronomy and Astrophysics, 341, 595

Glazunova, L. V., Yushchenko, A. V., Tsymbal, V. V., Mkrtichian, D. E., Lee, J. J., Kang, Y. W., Valyavin, G. G., \& Lee, B. C. 2008, The Astronomical Journal, 136, 1736

Goodricke, J. 1783, Royal Society of London Philosophical

Transactions Series I, 73, 474 
Greisen, E. W. 2003, Astrophysics and Space Science Lib., 285, 109

Gu, S. H., Tan, H. S., Shan, H. G., \& Zhang, F. H. 2002, Astronomy and Astrophysics, 388, 889

Güdel, M., Linsky, J. L., Brown, A., \& Nagase, F. 1999, ApJ, 511,405

Hartkopf, W. I., Mason, B. D., McAlister, H. A., Roberts, Lewis C., J., Turner, N. H., ten Brummelaar, T. A., Prieto, C. M., Ling, J. F., \& Franz, O. G. 2000, The Astronomical Journal, 119, 3084

Hartkopf, W. I., Mason, B. D., Wycoff, G. L., \& McAlister, H. A. 2010, Fourth Catalog of Interferometric Measurements of Binary Stars (Washington: U.S. Naval Obs.) http://ad.usno.navy.mil/wds/int4.html

Hatzes, A. P., Vogt, S. S., Ramseyer, T. F., \& Misch, A. 1996, The Astrophysical Journal, 469, 808

Hill, G., Barnes, J. V., Hutchings, J. B., \& Pearce, J. A. 1971 The Astrophysical Journal, 168

Hill, G., Perry, C. L., \& Khalesseh, B. 1993, A\&AS, 101, 579

Huenemoerder, D. P., Buzasi, D. L., \& Ramsey, L. W. 1989, The Astronomical Journal, 98, 1398

Lang, K. R., \& Willson, R. F. 1988, Astrophysical Journal, 328, 610

Lestrade, J. F., Mutel, R. L., Phillips, R. B., Webber, J. C., Niell, A. E., \& Preston, R. A. 1984, Astrophysical Journal, 282, L23

Lestrade, J.-F., Mutel, R. L., Preston, R. A., \& Phillips, R. B. 1988, Astrophysical Journal, 328, 232

Lestrade, J.-F., Phillips, R. B., Hodges, M. W., \& Preston, R. A. 1993, Astrophysical Journal, 410, 808

Lestrade, J. F., Preston, R. A., Jones, D. L., Phillips, R. B., Rogers, A. E. E., Titus, M. A., Rioja, M. J., \& Gabuzda, D. C. 1999, Astronomy and Astrophysics, 344, 1014

Lestrade, J. F., Rogers, A. E. E., Whitney, A. R., Niell, A. E., Phillips, R. B., \& Preston, R. A. 1990, Astronomical Journal, 99, 1663

Ma, C., Arias, E. F., Eubanks, T. M., Fey, A. L., Gontier, A.-M., Jacobs, C. S., Sovers, O. J., Archinal, B. A., \& Charlot, P. 1998, Astronomical Journal, 116, 516

Massarotti, A., Latham, D. W., Stefanik, R. P., \& Fogel, J. 2008, The Astronomical Journal, 135, 209

Massi, M., Menten, K., \& Neidhofer, J. 2002, Astronomy and Astrophysics, 382, 152

McAlister, H. A., Hartkopf, W. I., Hutter, D. J., \& Franz, O. G. 1987, The Astronomical Journal, 93, 688

Mutel, R. L., Doiron, D. J., Phillips, R. B., \& Lestrade, J. F. 1984, Astrophysical Journal, 278, 220

Mutel, R. L., Lestrade, J. F., Preston, R. A., \& Phillips, R. B. 1985, Astrophysical Journal, 289, 262

Mutel, R. L., Molnar, L. A., Waltman, E. B., \& Ghigo, F. D. 1998, Astrophysical Journal, 507, 371

Ottmann, R., \& Schmitt, J. H. M. M. 1996, Astronomy and Astrophysics, 307, 813

Pan, X., Shao, M., \& Colavita, M. M. 1993, Astrophysical Journal, 413, L129
Perryman, M. A. C., Lindegren, L., Kovalevsky, J., Hoeg, E., Bastian, U., Bernacca, P. L., Crz, M., Donati, F., Grenon, M., van Leeuwen, F., van der Marel, H., Mignard, F., Murray, C. A., Le Poole, R. S., Schrijver, H., Turon, C., Arenou, F., Froeschl, M., \& Petersen, C. S. 1997, Astronomy and Astrophysics, 323, L49

Peterson, W. M., Mutel, R. L., Güdel, M., \& Goss, W. M. 2010, Nature, 463, 207

Petrov, L. 2007, Astronomy and Astrophysics, 467, 359

Petrov, L., Gordon, D., Gipson, J., MacMillan, D., Ma, C., Fomalont, E., Walker, R. C., \& Carabajal, C. 2009, Journal of Geodesy, 83, 859

Pradel, N., Charlot, P., \& Lestrade, J. F. 2006, Astronomy and Astrophysics, 452, 1099

Press, W. H., Teukolsky, S. A., Vetterling, W. T., Flannery, B. P., Teukolsky, S. A., Vetterling, W. T., \& Flannery, B. P. 1992, Numerical recipes in FORTRAN. The art of scientific computing, ed. W. H. Press

Reid, M. J., Menten, K. M., Zheng, X. W., Brunthaler, A., \& Xu, Y. 2009, The Astrophysical Journal, 705, 1548

Reid, M. J., Readhead, A. C. S., Vermeulen, R. C., \& Treuhaft, R. N. 1999, The Astrophysical Journal, 524, 816

Retter, A., Richards, M. T., \& Wu, K. 2005, Astrophysical Journal, 621, 417

Richards, M. T., \& Albright, G. E. 1993, The Astrophysical Journal Supplement Series, 88, 199

Richards, M. T., Waltman, E. B., Ghigo, F. D., \& Richards, D. S. P. 2003, Astrophysical Journal Supplement Series, 147, 337

Ros, E., Marcaide, J. M., Guirado, J. C., Sardón, E., \& Shapiro, I. I. 2000, Astronomy and Astrophysics, 356, 357

Ros, E., \& Massi, M. 2007, Memorie della Societa Astronomica Italiana, 78

Ros, E., \& Reid, M. 2005, in Future Directions in High Resolution Astronomy, ed. J. Romney, Vol. 340, Astronomical Society of the Pacific, 482

Rosario, M. J., Mekkaden, M. V., \& Raveendran, A. V. 2008 , Information Bulletin on Variable Stars, 5836

Schmitt, J. H. M. M., \& Favata, F. 1999, Nature, 401, 44

Schuessler, M., \& Solanki, S. K. 1992, Astronomy and Astrophysics, 264, L13

Sjouwerman, L. O., Taylor, G. B., \& Benson, J. M. 2004, Bulletin of the American Astronomical Society, 205, 1605

Snajdrova, K., Boehm, J., Willis, P., Haas, R., \& Schuh, H. 2006, Journal of Geodesy, 79, 613

Stein, J. W., \& Beardsley, W. R. 1977, Vistas in Astronomy, 21, 43

Stern, R. A., Lemen, J. R., Schmitt, J. H. M. M., \& Pye, J. P. 1995, The Astrophysical Journal, 444, L45

Thompson, A. R., Moran, J. M., \& Swenson, G. W. 1986, Interferometry and synthesis in radio astronomy, ed. Thompson, A. R., Moran, J. M., \& Swenson, G. W.

Tokovinin, A., Thomas, S., Sterzik, M., \& Udry, S. 2006, Astronomy and Astrophysics, 450, 681

Torricelli-Ciamponi, G., Franciosini, E., Massi, M., \& Neidhoefer, J. 1998, Astronomy and Astrophysics, 333, 970

Trigilio, C., Leto, P., \& Umana, G. 1998, Astronomy and Astrophysics, 330, 1060

Vogt, S. S., \& Hatzes, A. P. 1991, IAU Colloq. 130: The Sun and Cool Stars. Activity, Magnetism, Dynamos, 380

Zavala, R. T., Hummel, C. A., Boboltz, D. A., Ojha, R., Shaffer, D. B., Tycner, C., Richards, M. T., \& Hutter, D. J. 2010, The Astrophysical Journal, 715, L44

Zombeck, M. V. 1990, Handbook of space astronomy and astrophysics, ed. Zombeck, M. V. 